\title{
Docetaxel als therapeutische Option für die adjuvante Chemotherapie des nicht-kleinzelligen Bronchialkarzinoms
}

\section{Docetaxel - a Therapeutic Option for Adjuvant Chemotherapy in NSCLC}

\author{
W. Eberhardt \\ Innere Klinik und Poliklinik (Tumorforschung), Universitätsklinikum Essen
}

$D$ ie E rgebnisse alleiniger L okaltherapien beim nicht-fernmetastasierten nicht-kleinzelligen B ronchialkarzinom (NSCLC) sind bisher insgesamt unbefriedigend. Auch in den frühen Stadien versterben die meisten Patienten innerhalb von 5 Jahren an ihrer E rkrankung (Tab. 1). D ies zu ändern, sollte ein wesentliches Ziel der Entwicklung neuer Therapiestrategien bei diesen Tumoren sein. D er nächste Schritt in der A nwendung der klinisch so aktiven Substanz Docetaxel beim NSCLC dürfte deshalb in einem $E$ insatz innerhalb von adjuvanten und neoadjuvanten Therapieprotokollen liegen. $\mathrm{H}$ ierbei ist bemerkenswert, dass die Taxane in der Therapie des NSCL C zu den wenigen Substanzen zählen, die auch nach Vorbehandlung mit einer Platintherapie noch objektive klinische R emissionen mit akzeptablen Ü berlebensraten erzielen können.

\section{(Neo)adjuvante Therapie im Stadium I bis III}

B is zum Stadium IIb gilt heute die alleinige Operation als Standardbehandlung des NSCLC, doch der Stellenwert einer adjuvanten, postoperativen Chemotherapie wird gerade in diesen frühen Stadien derzeit umfassend geprüft. Wenn der Wirkungsgrad unserer systemischen Behandlung durch die Integration der Chemotherapie in diesen frühen Stadien zunimmt, wird man auch erneut die Frage nach dem Stellenwert einer zusätzlichen R adiotherapie in diesen Stadien stellen müssen. A ufgrund der negativen E rgebnisse einer unlängst publizierten Metaanalyse zur adjuvanten Radiotherapie ist diese im Augenblick bei diesen Tumoren etwas in den $\mathrm{H}$ intergrund getreten. In den Stadien III a und III b hat allerdings die D urchführung einer zytostatischen Chemotherapie zusätzlich zu den Lokaltherapien Operation (überwiegend IIIa) oder $\mathrm{R}$ adiotherapie (überwiegend $\mathrm{III}$ b) bereits ihre klare B erechtigung gefunden.

In den randomisierten Studien zur neoadjuvanten Chemotherapie im Stadium IIIa [1-5] konnte relativ deutlich gezeigt werden, dass sich der biologische Verlauf der Tumoren durch eine Integration systemischer Therapiemaßnahmen verbessern lässt.
Tab. 1. Prognose des NSCLC nach klinischen Stadien [mod. nach 10]

\begin{tabular}{lll}
\hline Stadium & TNM & 5-Jahres-Ü berleben, \% \\
\hline Ia & T 1 N 0 & 60 \\
Ib & T 2 N O & 40 \\
II a & T 1 N 1 & 35 \\
II b & T 2 N 1, T 3 N 0 & 25 \\
III a & T 3 N 1, T 1-3 N & 15 \\
III b & T N 3, T 4 N & 5 \\
\hline
\end{tabular}

In einer $\mathrm{R}$ eihe von großen randomisierten Phase-III-Studien wird derzeit die W irksamkeit einer adjuvanten Chemotherapie beim NSCLC in den Stadien Ib bis (frühes, minimales) IIIa geprüft, wobei verschiedene R egime und Substanzen zum E insatz kommen. A llen diesen adjuvant applizierten Kombinationen ist gemeinsam, dass sie Platin als integralen Bestandteil aufweisen; häufig in einer K ombination mit $\mathrm{N}$ avelbine. A ktuell ist als die umfangreichste dieser $U$ ntersuchungen die aus Paris geleitete französische IA LT-Studie mit insgesamt 3300 Patienten zu nennen [6].

Auch in Kombination mit einer Radiotherapie hat sich die $\mathrm{G}$ abe einer zytostatischen Kombinationschemotherapie im Stadium IIIa/b als sinnvoll gezeigt, wie in den vier randomisierten Studien zum A usdruck kommt, die in Tabelle 2 kurz zusammengefasst werden. Die Integration einer Systemtherapie führt demnach zu signifikant besseren $L$ angzeitüberlebensraten der Patienten.

\section{Können wir in Zukunft auf Platin verzichten?}

$\mathrm{H}$ eute muss allerdings die Frage gestellt werden, ob wir Platin angesichts der Vielzahl neuer, hoch aktiver Substanzen beim NSCL C überhaupt noch benötigen. G epoolte $D$ aten aus vier aktuellen Phase-III-Studien, in denen jeweils Cisplatin alleine mit der entsprechenden Kombination von Cisplatin mit einer neuen Substanz verglichen wurde, belegen für Cisplatin als

\begin{tabular}{ll}
\hline KARGER & @ 2000 S. Karger G mbH , Freiburg \\
$\begin{array}{l}\text { Fax }+497614520714 \\
\begin{array}{l}\text { E-mail Information@K arger.de } \\
\text { www.karger.com }\end{array}\end{array}$ & $\begin{array}{l}\text { A ccessible online at: } \\
\text { www.karger.com/journals/onk }\end{array}$ \\
&
\end{tabular}

Dr. med. Wilfried Eberhardt

Innere U niversitätsklinik (Tumorforschung)

H ufelandstraße 55, D -45147 E ssen (G ermany)
und

Tel. +49 201 723-2168, Fax - 3131

E-mail wilfried.eberhardt@uni-essen.de 
Tab. 2. NSCL C III a/b - R adiotherapie versus Chemotherapie + sequentielle $\mathrm{R}$ adiotherapie in klinischen Studien

\begin{tabular}{|c|c|c|c|c|c|c|}
\hline Autor & $\mathrm{N}$ & $\begin{array}{l}\text { Chemotherapie } \\
\text { Gy }\end{array}$ & $\begin{array}{l}\text { R adiotherapie } \\
\text { M onate }\end{array}$ & $\begin{array}{l}\text { M edianes } \\
\text { Ü berleben } \\
\%\end{array}$ & $\begin{array}{l}\text { 5-Jahres- } \\
\text { Ü berleben } \\
\%\end{array}$ & $p$ \\
\hline Dillman & $\begin{array}{l}78 \\
77\end{array}$ & $\begin{array}{l}2 \times \mathrm{PV} \mathrm{bl} \\
2 \times \mathrm{PV} \mathrm{bl}\end{array}$ & $\begin{array}{l}60 \\
60\end{array}$ & $\begin{array}{r}13,7 \\
9,6\end{array}$ & $\begin{array}{r}17 \\
6\end{array}$ & $<0,05$ \\
\hline Le Chevalier & $\begin{array}{l}176 \\
177\end{array}$ & $2 \times \mathrm{PVCyCc}$ & $\begin{array}{l}65 \\
65\end{array}$ & $\begin{array}{l}12,0 \\
10,0\end{array}$ & $\begin{array}{l}6 \\
3\end{array}$ & $<0,02$ \\
\hline Sause & $\begin{array}{l}164 \\
163 \\
163\end{array}$ & $2 \times \mathrm{PVbl}$ & $\begin{array}{l}60 \\
60 \\
69,6\end{array}$ & $\begin{array}{l}13,7 \\
11,4 \\
12,2\end{array}$ & $\begin{array}{l}8 \\
5 \\
6\end{array}$ & $<0,04$ \\
\hline M attson & $\begin{array}{l}119 \\
119\end{array}$ & CAP & $\begin{array}{l}55 \\
55\end{array}$ & $\begin{array}{l}10,9 \\
10,2\end{array}$ & $\begin{array}{r}11 \\
7\end{array}$ & n.s. \\
\hline
\end{tabular}

M onotherapie eine sehr geringe $\mathrm{R}$ emissionsrate von im M edian $13,4 \%$. Neuere Substanzen wie D ocetaxel, Paclitaxel, G emcitabin, V inorelbin oder I rinotecan haben hier deutlich bessere Resultate mit Remissionsergebnissen zwischen 20 und 30\% erzielen können, allerdings sind bei diesen fortgeschrittenen Tumorstadien bei den Überlebenszeiten in den einzelnen A rmen keine signifikanten U nterschiede festzustellen (Tab. 2). E ine wirksame remissionsinduzierende Chemotherapie ist aber gerade in der adjuvanten Therapiesituation außerordentlich wichtig. In einer multizentrischen Phase-II-U ntersuchung setzten $M$ attson et al. [7] D ocetaxel als neoadjuvante Therapie bei 274 N SCLC-Patienten in den Stadien IIa bis IIIb ein. Je nach A usgangsstadium wurde eine Remissionsrate zwischen 21 (IIIb) und 37\% (IIb-IIa/T 3) erzielt. D ieA utoren haben eine derzeit noch laufende - Phase-III-Studie angeschlossen, in der 3 Zyklen Docetaxel $\left(100 \mathrm{mg} / \mathrm{m}^{2}\right)$ zum E insatz kamen, und wo die systemische I nduktionsbehandlung von einer L okaltherapie (O peration und/oder Strahlentherapie) gefolgt wurden.

\section{Docetaxel/Cisplatin neoadjuvant}

D ie wichtigsten Daten zur neoadjuvanten Therapie mit der Kombination aus D ocetaxel/C isplatin hat bisher eine Phase-IIStudie (SA KK) von Betticher et al. [8] an 34 Patienten im NSCLC-Stadium IIIa-N 2 geliefert. A $m$ Tag 1 und 2 erhielten die Patienten $40 \mathrm{mg} / \mathrm{m}^{2}$ Cisplatin, am Tag 1 zusätzlich $85 \mathrm{mg} / \mathrm{m}^{2}$ D ocetaxel. D ie beobachtete objektive R emissionsrate lag bei $66 \%$, eine R 0-R esektion wurde nach A bschluss der Induktionsbehandlung bei $70 \%$ der Patienten möglich. R osell und M itarbeiter prüfen derzeit in einer Phase-II-Studie den Stellenwert einer neoadjuvanten Therapie mit Cisplatin $\left(75 \mathrm{mg} / \mathrm{m}^{2}\right.$ am Tag 1), D ocetaxel (20 mg/m² am Tag 1, 8 und 15) und Gemcitabin (1000 mg/m am Tag 1 und 8 ) bei Patienten mit primär inoperablem Tumor im Stadium III a und III b.

\section{Prä- oder postoperative Chemotherapie?}

U nklar ist bisher allerdings noch völlig, ob es effektiver ist, die Chemotherapie als sogenannte Induktionstherapie vor der O peration zu applizieren oder adjuvant im A nschluss an die O peration durchzuführen.
A Is Vorteile der postoperativen Chemotherapie könnten sich erweisen, dass frühzeitig operiert werden kann, dass der Zeitraum einer möglichen metastatischen Streuung kürzer bleibt und dass das R isiko für perioperative Komplikationen meist nicht erhöht wird. A Is N achteile einer solchen postoperativen Chemotherapie fallen allerdings ins $\mathrm{G}$ ewicht, dass erfahrungsgemäß die B ereitschaft der Patienten zu einer postoperativen Chemotherapie deutlich geringer ist, dass die D osisintensität der Chemotherapie postoperativ deutlich zurückgenommen werden muss und dass die operativen $\mathrm{E}$ ingriffe möglicherweise nicht so organschonend vorgenommen werden können.

D ie präoperative Chemotherapie kann hier nun den Vorteil einer frühzeitigen systemischen Therapiemöglichkeit bieten, die therapeutische Reduktion des Tumorvolumens vor der L okaltherapie und die sehr frühe B ehandlung einer möglichen M ikrometastasierung. D ie D osisintensität der Chemotherapieprotokolle kann präoperativ deutlich höher liegen als postoperativ und dies lässt sich auch in einer besseren TherapieCompliance bei den Patienten ablesen. I $m$ A Ilgemeinen stehen die Patienten eine aggressive Induktions-Chemotherapie psychisch viel besser durch, wenn als «Licht am Ende des Tunnels» eine O peration vorgesehen ist. A uf der anderen Seite führt eine präoperative A pplikation der Chemotherapie zu einer gering höheren perioperativen M orbidität, vor allem zu einem erhöhten $\mathrm{R}$ isiko von kardiovaskulären Komplikationen und $\mathrm{E}$ reignissen. $\mathrm{H}$ ierbei ist zu berücksichtigen, dass die meisten Patienten bereits durch den langjährigen N ikotinabusus kardiovaskulär deutlich vorgeschädigt sind. B ei Patienten mit primär progressiver E rkrankung unter der Induktions-C hemotherapie geht unter U mständen die O perabilität verloren.

M ögliche Weiterentwicklungen in der Zukunft sind wöchentliche A pplikationsformen von D ocetaxel (z. B. auch Tag-1- und -8-R egime), platinhaltige K ombinationen sowie durchaus auch platinfreie $Z$ weier- oder $D$ reierkombinationen in hoher $D$ osisdichte (z.B. D ocetaxel/G emcitabin).

\section{Chemoradiotherapie plus/minus Operation potentiell kurativ}

In Essen wird schon seit A nfang 1991 mit guten E rgebnissen eine präoperative Chemostrahlentherapie (Cisplatin/E toposid) in den primär inoperablen Stadien III a und III b eingesetzt. E S 
Abb. 1 Ü berlebenszeit: R 0-R esektion ( $\longrightarrow$ versus keine R 0-R esektion (-).

(E ssener Studie der Thoraxonkologie-G ruppe, mod. nach E berhardt [9]).

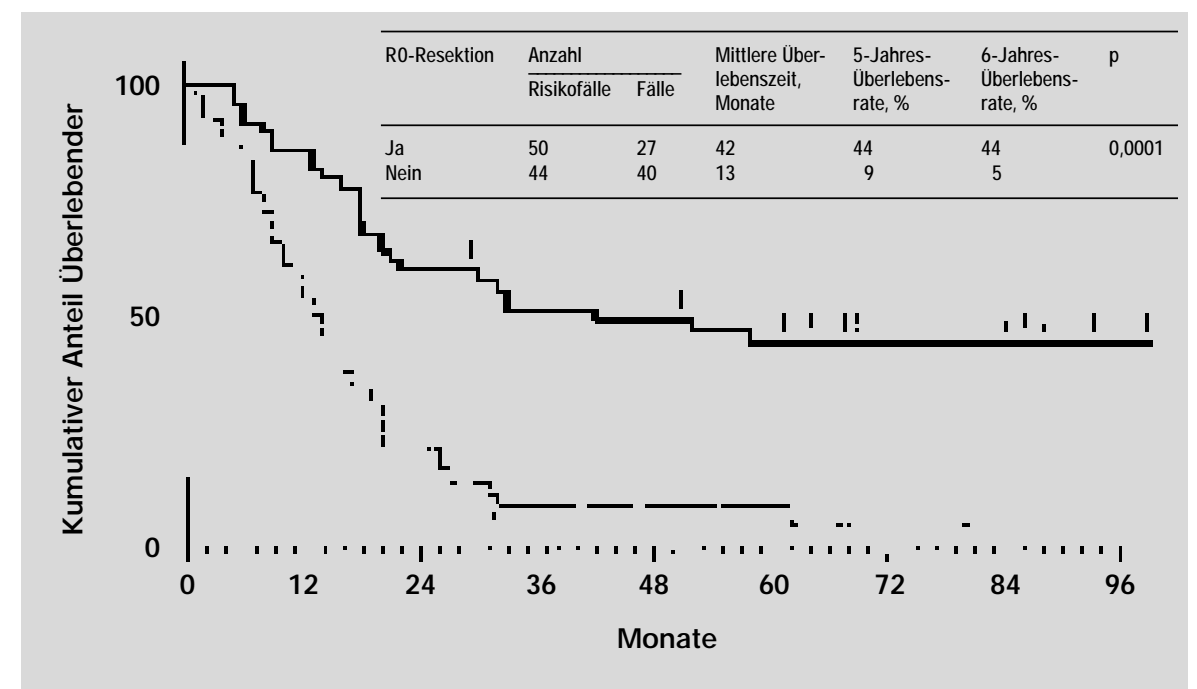

handelt sich dabei um eine potentiell kurative Therapieoption, wie der L angzeitverlauf mit einer 5-Jahresüberlebensrate von fast $50 \%$ bei Patienten nach durchgeführter kompletter Tumorresektion (R 0) zweifelsfrei belegt (A bb. 1) [9]. Durch die Integration neuer Substanzen in die Induktions-Chemotherapie kann dieses K onzept möglicherweise noch weiter optimiert werden (höhere E ffektivität? geringere Toxizität?). Wir konnten mit der Kombination aus Cisplatin und Paclitaxel in dieser Induktionsphase bereits erste positive Erfahrungen sammeln. A Is Konsequenz aus diesen E rgebnissen planen wir, die Kombination aus Docetaxel und Cisplatin ebenfalls als Induktions-Chemotherapie innerhalb einer prospektiven Phase-I/II-Studie in diesen Therapiestadien zu testen.

\section{Zusammenfassung}

D er adjuvante und neoadjuvante $E$ insatz von $D$ ocetaxel in den Stadien I bis III des NSCLC sollte in den nächsten Jahren weiter im $\mathrm{R}$ ahmen von klinischen Studien geprüft werden, vor allem in Kombination mit Platinderivaten. Bisher ist eine Kombination von D ocetaxel mit Platinderivaten am besten für die neoadjuvante Therapie im Stadium III untersucht worden. $M$ öglicherweise bieten nicht-platinhaltige Kombinationen mit D ocetaxel - z.B. mit Gemcitabin oder Vinorelbin - hier die zusätzliche M öglichkeit, die Toxizität bei zumindest vergleichbarer E ffektivität zu reduzieren. D er E insatz von D reierkombinationen könnte unter U mständen sogar noch wirksamer sein, wenn allerdings die Toxizität solcher «Triplets» nicht zu ausgeprägt ist. E in wichtiger Schritt in den nächsten Jahren wird deshalb sein, diese Phase-I - und -II-Studien beim NSCLC rasch durchzuführen, um danach die konsekutiven großen Phase-III-U ntersuchungen anschließen zu können.

\section{Literatur}

1 Pass HI, Pogrebniak H W, Steinberg SM, M ulshine I, $M$ inna J: R andomized trial of neoadjuvant therapy for lung cancer: interim analysis. A nn Thorac Surg 1992;53:992-998.

$2 \mathrm{R}$ oth, JA , Fossella FV, Komaki $\mathrm{R}$, et al: A randomized trial comparing preoperative chemotherapy and surgery with surgery alone in resectabel stage IIIA non-small cell lung cancer. J Natl Cancer Inst 1994 86:673-680.

3 R oth JA, A tkinson EN, Fossella $F$, Komaki $R$, Bernadette R yan $M$, Putnam J B J r, L ee J S, D hingra $H$, De Caro L, Chasen M, Hong WK: Long-term follow-up of patients enrolled in a randomized trial comparing perioperative chemotherapy and surgery with surgery alone in resectable stage IIIA non-smallcell lung cancer. L ung Cancer 1998;21:1-6.

4 Rosell R, Gomez-Codina J, Camps C, et al: A randomized trial comparing preoperative chemotherapy plus surgery with surgery alone in patients with nonsmall cell lung cancer. N Engl J Med 1994;330: 153-158.

5 R osell R, G omez-Codina J, Camps C, Javier Sanchez J, M aestre J, Padilla J, Canto A, A bad A, R oig J: Preresectional chemotherapy in stage IIIA non-smallcell lung cancer:A 7-year assessment of a randomized controlled trial. L ung Cancer 1999:26:7-14.

$6 \mathrm{~L}$ e Chevalier T, A rriagada R, Q uoix E, Ruffie $P_{\text {, }}$ $M$ artin $\mathrm{V}$, Tarayre $\mathrm{M}: \mathrm{R}$ adiotherapy alone versus combined chemotherapy and radiotherapy in unresectable non-small cell lung carcinoma.J $\mathrm{N}$ atl $\mathrm{C}$ ancer Inst 1991;83:417-423.

7 Mattson KV, Ten Velde G, Krofta K, Tonelli $D$, A rmellini J, A bratt $R$ : Taxotere as neoadjuvant therapy for radically-treatable stage III NSCLC. Preliminary results of an international phase III study. ProcA m Soc Clin O ncol 2000;19(abstr 1890).
8 B etticher DC, H su Schmitz SF, Gauthier Y, Von B riel C, B reitenbücher A, R oth A, Spiliopoulos A, Pless M, Stahel R, Weder W, Tötsch M, Cerny T, R is HB: Neoadjuvant chemotherapy with cocetaxes (DOC, Taxotere) and cisplatin (CIS) in patients (pts) with non-small cell lung cancer (NSCLC), stage IIIA , N2 is highly active with few toxicities. Proc A m Soc Clin O ncol 1999;18(abstr 1802).

9 E berhardt W, Wilke $H$, Stamatis $M, H$ arstrick $A$, M enker $H$, K rause $B$, M üller M R, Stahl $M$, Flasshove $M, B$ udach $D, G$ reschuchna $D$, Konietzko $N$, Sack H, Seeber S: Preoperative chemotherapy followed by concurrent chemoradiation therapy based on hyperfractionated accelerated radiotherapy and definitive surgery in advanced NonSmall-Cell Lung Cancer: $M$ ature results of a phase II trial I Clin O ncol 1998;16:622-634.

10 M ountain CF. R evisions in the International System for Staging L ung Cancer. Chest 1997:111;1710-1727. 